\title{
Correcting J-R Curve Mismatch Twist with Normalization Analysis
}

J. D. LANDES and R. HERRERA

Department of Engineering Science and Mechanics, University of Tennessee, Knoxville, TN 37996, USA

\section{ABSTRACT}

Automatic methods for measuring crack length in a J-R curve fracture toughness test may have small errors which result in larger "mismatch twisting of the $R$ curve. A method for determining crack length from normalized load and displacement curves can be used to correct this problem. Examples are presented here showing the "mismatch twist" and the resulting correction. This error in J-R curve evaluation can give rise to several problems including incorrectly inferred size effects and unconservative values of the tearing modulus used for instability prediction. It is also a problem for the case where $\mathrm{R}$ curve extrapolation is necessary.

\section{KEYWORDS}

Fracture toughness; J-R curve; crack extension; normalization; mismatch twist.

INTRODUCTION

Ductile fracture toughness can be measured with the $\mathrm{R}$ curve where the crack Ductile fracture toughness can be measured with the $R$ curve where the crack extension $\triangle$ a is plon laboratory test a continuous measure measure measurement Several methods have been developed for indirect crack length measurement most commonly used is the elastic unloading compliance method (3) which has been incorporated into the ASTM standard test method for the J-R curve (E1152).

Elastic compliance measurement of crack length requires some sophisticated gages and recording equipment and can be a difficult task for the novice. Even for the experienced testor, crack length measurement by this method can produce error The standard test method allows 15 percent error in measurement of crack length change (comparing compliance measured crack length change to a physical chent on the fracture surface), but this error is often exceeded. 

curve, incorrect crack length measurement can influence the calculated value of or "mismatch twist".

The $R$ curve is often used for stability analysis where the slope is the important Thameter (4). An error in the R curve shape could cause a large error in this slope. parso $R$ curves dill laboratory specimens may not result in enough crack extension to analyze larger structures and extrapolation may be desired. mism extent will not give a correct final section of $R$ curve for extrapolation. mismatch twist will a correct final point by physically matching the The $R$ curve can final crack leng , can insure a correct final point and eliminate any $R$ curve twist displacement (5, 6) can mismatch twist due to mismate example results of this correction.

MISMATCH TWIST

the standard ASTM method E1152, where $J$ is plotted on the ordinate and crack extension, $\Delta a$, on the abscissa. An error in $\Delta$ a measurement directly changes the value on the on the however, the calculation contains crack length terms and plastic displacement terms which are also influenced by crack length. Therefore an incorrect $\Delta a$ measurement also changes the value of reduces $J$ whereas underestimate increases $J$. The result for a single point is show $J-R$ in Figure $1 \mathrm{a}$. An accumulation of incorrect curve as shown in Fig

slope significantly.

\section{NORMALIZED J-R CURVE EVALUATION}

method for evaluating the J-R curve form normalized load and displacement has been previously presented by the authors $(5,6,7)$. This method uses the relationships between load, plastic anships can be given as

suggested by Ernst (8). These re ationships where the displacement, $v$, is se arated $v_{p}$, which are related to bend specimens as

$$
P_{N}=\frac{P_{W}}{B b^{2} g(b / W)}=H\left(\frac{v_{p l}}{W}\right)
$$

政 Where $W$ is specimen width, b is uncracked ligarack length is given by equation 1. relationship between load, displacement and in Handbooks, therefore if the The compliance function $\mathrm{ClaW}$ ) is tabulated can be continuously determined plasticity function $h\left(P_{N}\right)$ is known, crack length by the authors was to assume a from values of $P$ and $V$. The method usents and determine constants at known functional form with some unknown constants and determing functional form of $h\left(P_{N}\right)$ values of crack length, namelyinitial and final values. The function a straight line

used most
equation.

The functional form of $h$ is shown graphically in Figure 2. Where normalized load 作 $P_{N}$ is plotted versus $(6,9)$ from

$$
J=J_{e l}+J_{p l}=\frac{K^{2}}{E}+b \eta g A_{N}
$$

where $E$ is effective elastic modulus $\eta$ is a function of $a / W$ used in $J$ calculation and $A_{N}$ is area under the normalized plot of Figure 2 . This calculation of $J$ can be made directly from known values of load, displacement and crack length and differs from the traditional I calculation which is made incrementally.

The direct determination of $J$ and the use of the final crack length in determining $h\left(P_{N}\right)$ causes the crack length to go through a final point which agrees with the physically measured crack length. Therefore, the initial and final parts of the $\mathrm{R}$ curve are correct when analyzed by the normalization method. The only questionable part lies in the center section where all crack length values are inferred by the relationship in equations 1 and 2. Numerous examples were checked where the $R$ curve determined from the normalization method was compared with the one determined from the compliance method (6). Examples where the compliance method predicted well the final measured crack length were used for comparison. A typical example illustrating the good agreement is shown in Figure 3. This shows a crack length difference of about 0.015 inch $(0.4$ $\mathrm{mm}$ ) which occurs in the middle of the $\mathrm{R}$ curve. Average differences were usually in

\section{MISMATCH CORRECTION}

Since the normalization procedure gives the most accurate determination of the $\mathrm{J}$ $R$ curve at the large $\Delta a$ values (where compliance seems to be most inaccurate), this method can be used to correct some of the compliance determined R curves. The mismatch occurs both in crack length and J values. Equation 3 shows the parameters used in the determination of the plastic component of $J$. From these paramethe length. The other parameters $g$ and $n$ vary weakly with crack length and do not contribute significantly. Figures 4 and 5 show some examples of $J-R$ curves where the final crack length was incorrectly predicted by compliance. In Figure 4 , compact specimen of width 1 inch $(25 \mathrm{~mm})$ had an error in final crack length of $22 \%$ with compliance overestimating the physical crack length. The top part of the figure shows the result for the normalized load and plastic displacement plot comparing compliance prediction with the normalized prediction. The bottom shows the resulting J-R curves. For this case, the over prediction of crack length resulted in an underprediction of ligament $b$ and an underprediction of normalized area $A_{N}$. The latter is largely due to the error in prediction of plastic displacement that results from incorrect crack length. The combined result of the two is a large underprediction of the final and a mismatch twist" of the final part of the $R$ curve that is great enough to causea nisme normalized method corrects this part of the $R$ curve and shows the correct continually increasing slope

Figure 5 shows another example of a compact specimen with $W=4$ inches (102 $\mathrm{mm}$ ). In this case, the final crack length was underpredicted by compliance as compared with the final measured value by $38 \%$. The resulting J-R curve Figure 5 shows the opposite result from Figure $4 b$ in that the compliance predicted $R$ curve take a final slope which is too large.

\section{MISMATCH CONSEQUENCE}

The examples shown in Figures 4 and 5 were both part of individual studies to examine effects of size on J-R curve (10). The error in the final crack length prediction led to some incorrect conclusions. The R curve example of figure 4 is 
plotted with a specimen of the same material but 20 times larger in Figure 6 . The conclusion here from the compliance evaluation of the R curve was that the smaller specimen had an apparent size effect at the larger values of crack extension. This was then corrected by introducing a new correlating parameter, the modified which eliminated the apparent size effect (11, 12). Hownal crack length. This is needed to correct this was an analysis to correct the final crack the apparent size provided by the normalization method. As shown in Figurion are used in the J-R effect is eliminate

The example in Figure 5 shows the effect of mismatch on the final $R$ curve slope.

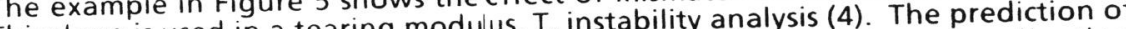
This slope is used in a tearing about 12 . The normalization tearing modulus from com the modulus of about 5 . this shows procedure was used to corre misual mismatch in an overestimate is to underpredict with the compliance estimate, as in this final crack long example, $R$ curve data to materials recount in part for uncorservative applications of $R$ curves to structura andysis.

An additional case where a correct final section of the J-R curve is essential is in the extrapolation of this curve. Very often the length of the R curve generated from the test specimen does not have sufficient crack extension for the intended application. Use of larger specimens is often not an alternative as the case of nuclear surveillance specimens where the specimen size is dictated by the size of the surveillance capsule, and an extrapolation of the $R$ curve is necessary. For an accurate extrapolation the final section of the $R$ curve musth method of normalization gives a correct final crack length which insusis for $R$ curve curve slope should be nearly correct. This then provides a good bi extrapolation.

\section{SUMMARY}

" caused by incorrectly predicting final crack length on a J-R The "mismatch twist caused by curve can cause several problems which are not correct. The normalized method modulus and apparent size final crack length and for evaluating the $J-R$ curve eliminates the error in final $J-R$ curves can be

eliminates this "mismatch an extrapolation of the $R$ curve is necessary.

\section{REFERENCES}

1. Landes, J and Begley, J A. "Recent Developments in JIC Testing," Develop 632 W F Brown $1 \mathrm{r}$ and I G Kaufman, Eds., American Society for Testing and Materials, 1977 pp $57-81$

2. Dawes Integral Concepts, “ Elastic-Plastic Fracture ASTM STP 668, J. D. Landes, . A. Begley, and G. A. Clarke, Eds., American Society for Testing and Materials, 1979, pp. 307-333.

3. Clarke, G. A Andrews, W. R., Paris, P. C., and Schmidt, D. W., "Single Specime Tests for J JC Determination" Mechanics of Crack Growth STP 590, American Society for Testing and Materlals, (Elastic-Plastic Fracture ASTM Tearing Mode of Elastic-Plasic Cack G. A. Clarke Eds, 1979, pp. 5-36
5. Herrera R and Landes, J. D. "A Direct J-R Curve Analysis of Fracture Toughness Tests," Journal of Testing and Evaluation, JTEVA, Vol. 11, No. 5, Sept. 1988, pp

6. Herrera, R. and Landes, J. D., “Direct J-R Curve Analysis: A Guide to the Mresented at the 21st Nation

7. Landes, J. D and Herrera, R. "A New Look at J-R Curve Analysis," International Journal of Fracture, Vol. 36: R9-R14, 1988

8. Ernst, H. A Paris, P. C. and Landes, J. D., "Estimations on J-Integral and Tearing Modulus T From a Single Specimen Test Record, Fracture Mechanics, Thirteenth Conference, ASTM STP 743, Richard Roberts, Ed., American Society for Testing and Materials, 1981, pp. 476-502.

9. Landes, J. D. and Herrera, R., "Calculation of J From Test Records for the Growing Crack, International Journal of Fracture, Vol. 36: R15-R20, 1988.

10. McCabe, D. E., Landes, J. D. and Ernst, H. A., "An Evaluation of the J-R Curve Method for Fracture Toughness Characterization, Elastic-Plastic Fracture Second Symposium, Vol. II - Fracture Resistance Curves and Engineering App

11. Landes $R$ et "Elastic-Plastic Methodology to Establish R Curves and Instability Criteria - Sixth Semiannual Report," EPRI Contract No. RP1238-2.

August 4, 1982.
2. Ernst, H. A." "Material Resistance and Instability Beyond J-Controlled Crack Growth," Elastic-Plastic Fracture: Second Symposium, Volume I - Inelastic Analysis, ASTM 803, C.F Shih and J.P. Gudas, Eds. 1983, pp. 1-191-1-213. 


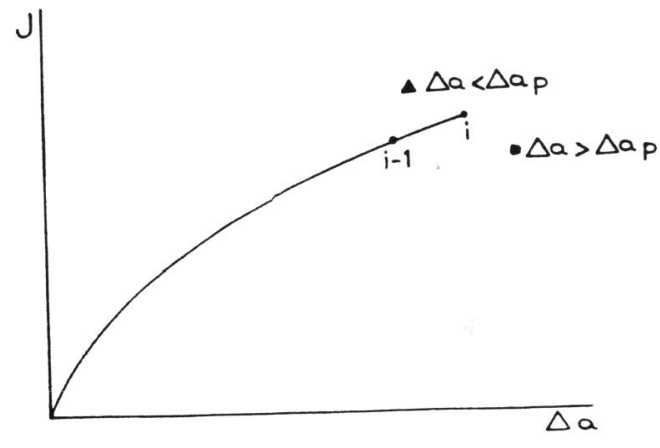

a) Single Point Mismatch

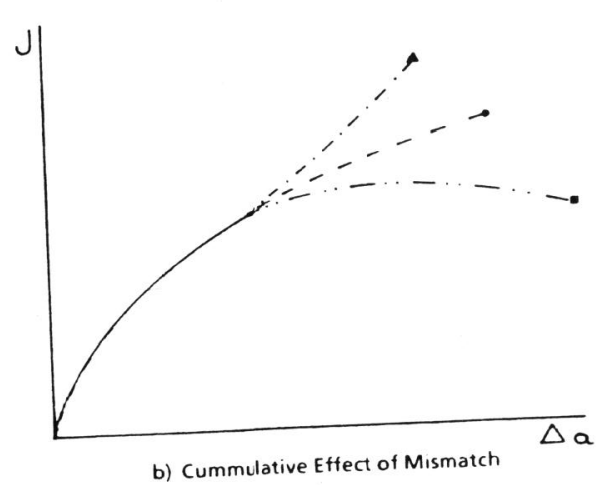

Figure 1 Schematic Effect of Mismatching Final $\Delta a$ on the J R Curve

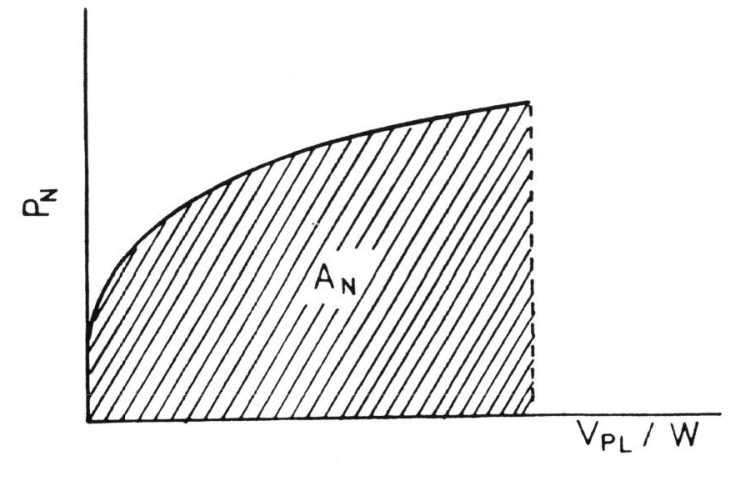

Figure 2 Normalized Load versus Plastic Displacement

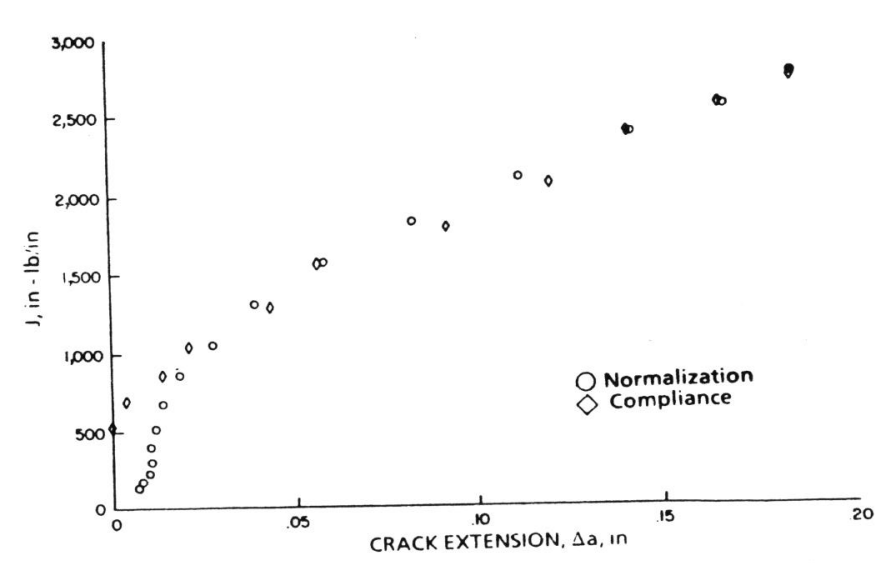

Figure 3 J-R Curve Normalization versus Compliance for A508 Steel 

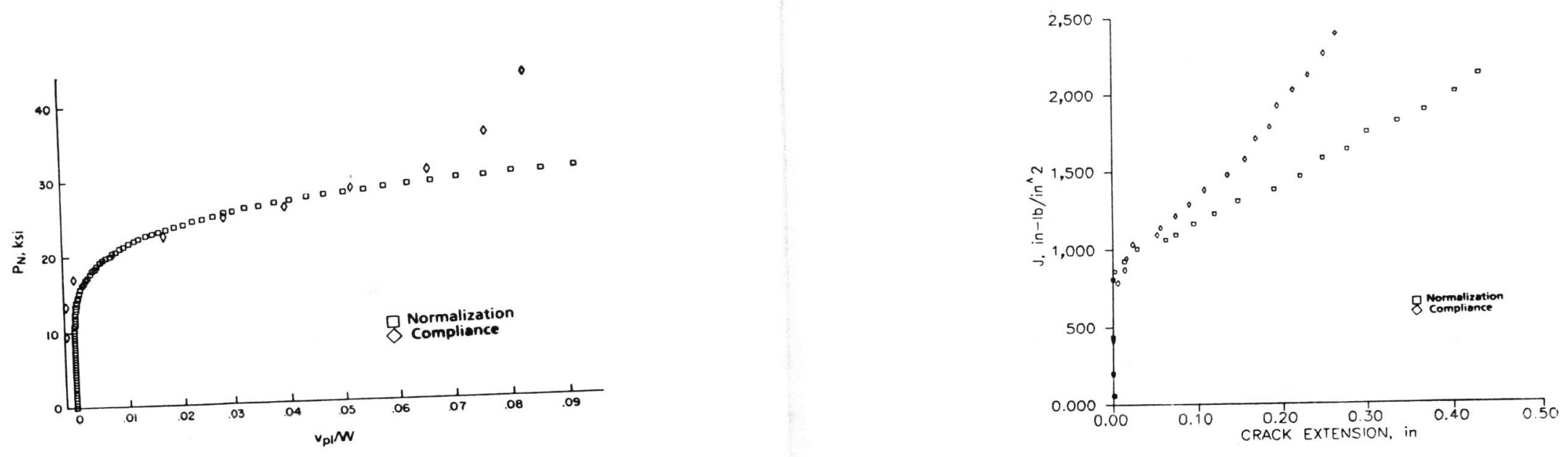

Figure 5 J-R Curve Normalization and Compliance Methods for a Ni Steel.
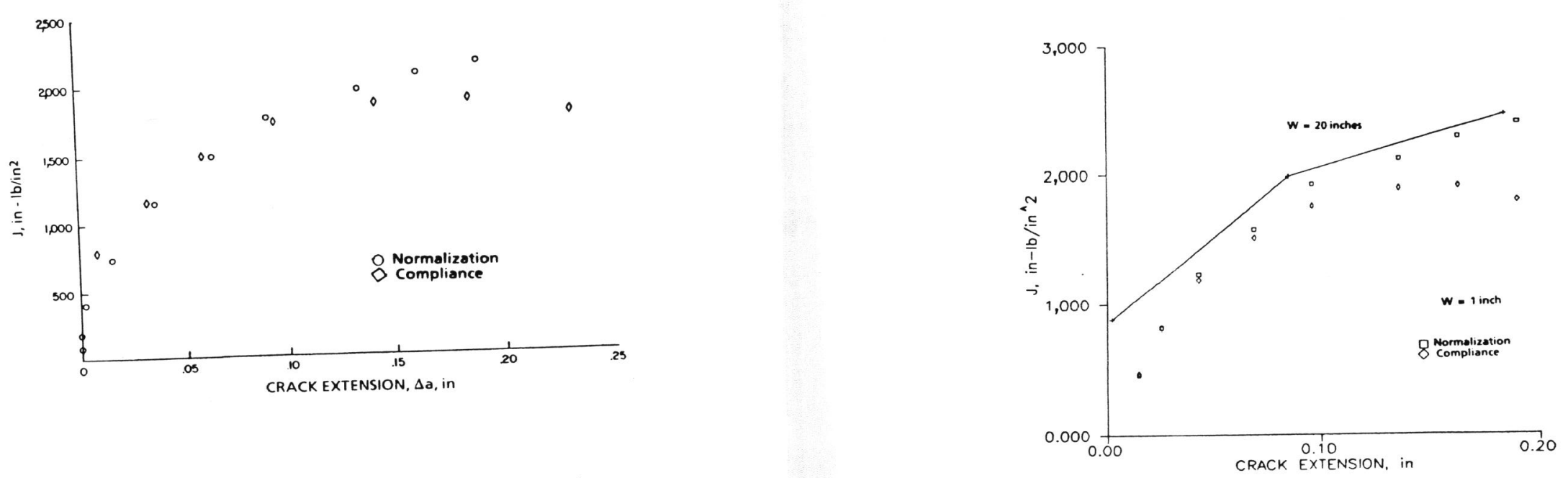

Figure 4 Normalized Load Versus Displacement (Top) and J-RCurve Normalization and Compliance (Bottom) for an A508 Steel.

Figure 6 J-R Curve Size Comparison for a Large (20 inches) and Small (1 inch) Specimen of A508 Steel. 